Article

\title{
Comparison of Anthraquinones, Iridoid Glycosides and Triterpenoids in Morinda officinalis and Morinda citrifolia Using UPLC/Q-TOF-MS and Multivariate Statistical Analysis
}

\author{
Maoyuan Wang ${ }^{1,2}$, Qinglong Wang ${ }^{1,2}$, Qing Yang ${ }^{1,2}$, Xiaoxia Yan ${ }^{1,2}$, Shixiu Feng ${ }^{3, *}$ \\ and Zhunian Wang ${ }^{1,2, *}$ \\ 1 Tropical Crops Genetic Resources Institute, Chinese Academy of Tropical Agricultural Sciences/Key \\ Laboratory of Crop Gene Resources and Germplasm Enhancement in Southern China, Ministry of \\ Agriculture, Haikou 571101, China; wmy81@163.com (M.W.); qlwang1983@163.com (Q.W.); \\ yangqinghnu@163.com (Q.Y.); yxx6232@163.com (X.Y.) \\ 2 Tropical Wild Plant Gene Resource, Ministry of Agriculture, Danzhou 571737, China \\ 3 Key Laboratory of South Subtropical Plant Diversity, Fairy Lake Botanical Garden, Shenzhen \& Chinese \\ Academy of Sciences, Shenzhen 518004, China \\ * Correspondence: fengshixiu@scbg.ac.cn (S.F.); 13807596560@163.com (Z.W.); Fax: +86-755-25702889 (S.F.); \\ +86-898-233006150 (Z.W.)
}

Received: 28 November 2019; Accepted: 30 December 2019; Published: 31 December 2019

\begin{abstract}
Roots of Morinda officinalis and Morinda citrifolia have been interchangeably used in traditional Chinese medicine. However, there is no experimental evidence to support this. In this study, a ultra-performance liquid chromatography quadrupole time-of-flight mass spectrometry (UPLC/Q-TOF-MS)-based approach and a multivariate statistical analysis (MSA) were adopted to compare the difference in the chemical compounds present in the root extract of $M$. officinalis and M. citrifolia. There were 26 anthraquinones, 15 triterpenes, and 8 iridoid glycosides identified in the root extracts of $M$. officinalis, 30 anthraquinones, 1 triterpene, and 8 iridoid glycosides in the root extracts of $M$. citrifolia. Among these, 25 compounds presented in both plants. In addition, a principal component analysis (PCA) showed that these two herbs could be separated clearly. Furthermore, an orthogonal partial least squares-discriminant analysis (OPLS-DA) found 9 components that could be used as chemical markers to discrimination the root extracts of M. officinalis and M. citrifolia. In addition, the results of a Cell Counting Kit 8 (CCK-8) assay and cell colony formation assay indicated that methanol root extracts of $M$. officinalis and $M$. citrifolia showed no cell cytotoxicity to normal cells, even promoted the proliferation of normal liver cells. To our knowledge, this is the first time that the differences between the root extracts of M. officinalis and M. citrifolia (Hainan province) have been observed systematically at the chemistry level.
\end{abstract}

Keywords: Morinda officinalis; Morinda citrifolia; UPLC/Q-TOF-MS; multivariate statistical analysis

\section{Introduction}

Morinda officinalis (M. officinalis) and Morinda citrifolia (M. citrifolia) belong to the family Rubiaceae [1] The roots of $M$. officinalis have long been used ass traditional medicine for the strengthening of bones and immunity, and the nourishment of the kidneys [2]. It has also been used to treat impotence, osteoporosis, depression and inflammatory diseases, such as rheumatoid arthritis and dermatitis [2]. Moreover, the clinical effects of $M$. officinalis have been proved by modern pharmacological studies [3-6]. The fruit of M. citrifolia is also called Noni in Hawaii and the juice made of it has been used as 
alternative medicine for arthritis, high blood pressure, diabetes, mental depression, sprains, and muscle aches [7-9]. The M. citrifolia fruit extract has been shown to exhibit antimicrobial, antifungal, antioxidant, anti-arthritic, and anticancer activities [10,11]. Furthermore, it also shows anxiolytic and sedative activities, and immunity enhancing activity $[12,13]$. M. officinalis and M. citrifolia were commonly distributed in the south of China (Guangdong, Guangxi and Hainan province) and are named as "HAIBAJI" and "BAJITIAN". In Hainan province, both plants are surprisingly called BAJI, and their roots have been used as edible herbal plants to treat sexual impotence, spermatorrhea, irregular menstruation, and female infertility [14]. However, evidence for such uses remains elusive, although both plants contain anthraquinones and iridoid glycosides $[1,15]$. In this study, we aim to determine whether the roots of the two plants have the same active chemical constituents in terms of their pharmaceutical functions. Quadrupole time-of-flight mass spectrometry (Q/TOF-MS) coupled with Ultra-performance liquid chromatography (UPLC) can be comprehensively used to analyze unknown compounds in an organism [16]. Hence, this study examines the principal chemical constituents of the roots of both species using UPLC/QTOF-MS coupled with a multivariate statistical analysis, and subsequently the maker components were elucidated by a principal component analysis (PCA).

\section{Results and Discussion}

\subsection{Identifying the Chemical Constituents of Morinda officinalis (M. officinalis) and Morinda citrifolia (M. citrifolia) Based on UPLC/Q-TOF-MS/MS}

The total ion chromatogram (TIC) in the negative ion mode of M. officinalis and M. citrifolia root extracts is illustrated in Figure 1. The information of identified compounds is summarized in Table 1. Compounds 1-8 were identified as iridoid glycosides, and their structures are displayed in Figure 2. Generally, this class of compounds underwent a loss of a glucose unit (162) to yield a fragment (M-162) and a subsequent elimination of $44\left(\mathrm{CO}_{2}\right)$ and/or $28(\mathrm{CO})$ could occur after the loss of glucose unit (Compounds 1 and 3). Compounds 5, 6, and 8 easily gave fragment ions of a glucoside (180), $\mathrm{C}_{2} \mathrm{H}_{2} \mathrm{O}(42)$, and $\mathrm{COOH}$ (45) produced from the deprotonated ion [17-19]. The proposed fragmentation pathway is shown in Figure $\mathrm{S} 1$.

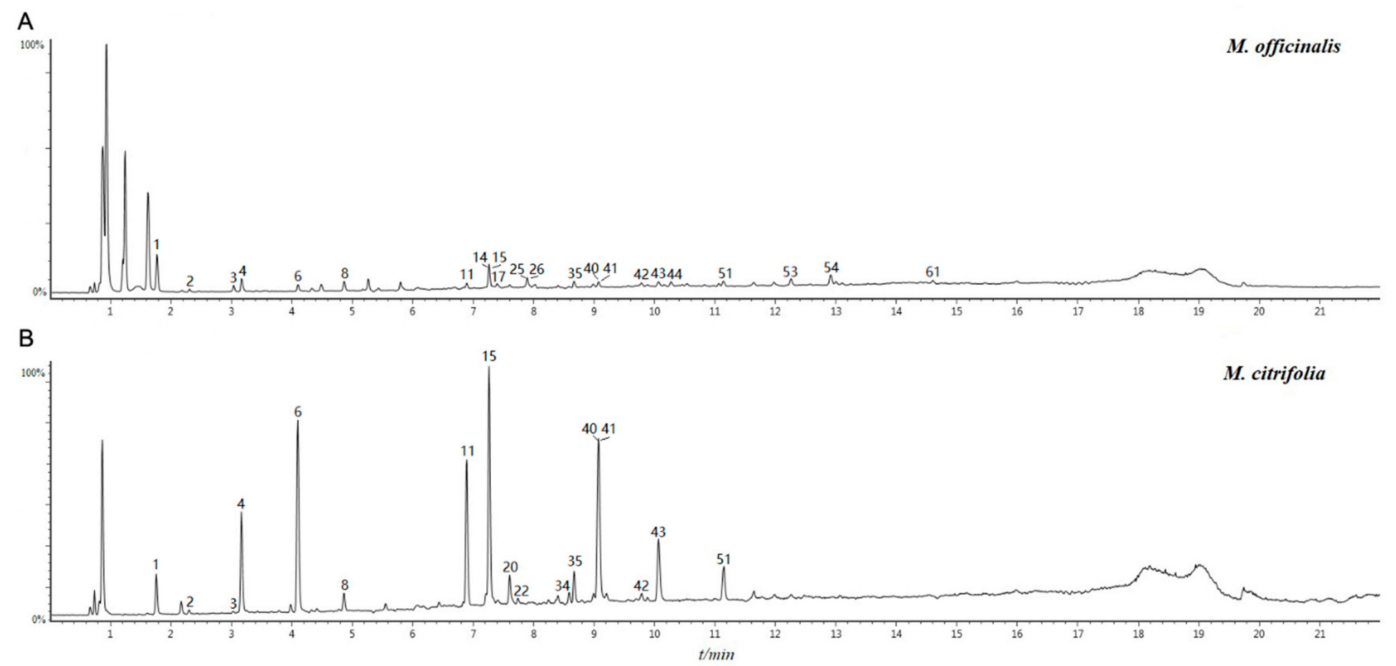

Figure 1. The total ion chromatogram (TIC) in the negative ion mode of samples: A for methanol extract of roots of $M$. officinalis roots, B for methanol extract $M$. citrifoli roots. 
Table 1. The mass spectrometry data and identified chemical compounds from M. officinalis and M. citrifolia.

\begin{tabular}{|c|c|c|c|c|c|c|c|c|c|c|c|}
\hline \multirow{2}{*}{$\begin{array}{c}\text { Peak } \\
\text { No. }\end{array}$} & \multirow{2}{*}{$\mathbf{R t}^{\mathrm{e}}(\min )$} & \multirow{2}{*}{ Formula } & \multirow{2}{*}{ Quasi-Molecular Ion } & \multirow{2}{*}{ MS $^{2}$ Ions } & \multicolumn{2}{|c|}{ Molecular Weight } & \multirow{2}{*}{$\begin{array}{c}\text { Mass } \\
\text { Error (ppm) }\end{array}$} & \multirow{2}{*}{ Identification } & \multirow{2}{*}{ Type } & \multicolumn{2}{|c|}{ Source } \\
\hline & & & & & Observed & Theoretical & & & & M. O & M. C \\
\hline 1 & 1.76 & $\mathrm{C}_{16} \mathrm{H}_{22} \mathrm{O}_{11}$ & $389.1087[\mathrm{M}-\mathrm{H}]^{-}$ & $\begin{array}{l}209.0457 \\
137.0610\end{array}$ & 390.1159 & 390.1162 & -0.7 & monotropein & I & $\sqrt{ }$ & $\sqrt{ }$ \\
\hline 2 & 2.30 & $\mathrm{C}_{16} \mathrm{H}_{22} \mathrm{O}_{11}$ & $389.1089[\mathrm{M}-\mathrm{H}]^{-}$ & - & 390.1162 & 390.1162 & -0.1 & diacetylasperulosidic acid & $\mathrm{I}$ & $\sqrt{ }$ & $\sqrt{ }$ \\
\hline 3 & 3.03 & $\mathrm{C}_{16} \mathrm{H}_{22} \mathrm{O}_{10}$ & $419.1191\left[^{\mathrm{M}}+\mathrm{HCOO}^{-}\right.$ & $\begin{array}{l}211.0613 \\
123.0454\end{array}$ & 374.1209 & 374.1213 & -1.0 & geniposidic acid & I & $\sqrt{ }$ & $\sqrt{ }$ \\
\hline 4 & 3.17 & $\mathrm{C}_{18} \mathrm{H}_{24} \mathrm{O}_{12}$ & $477.1248\left[\mathrm{M}+\mathrm{HCOO}^{-}\right.$ & $\begin{array}{l}417.1039 \\
371.0983 \\
209.0453 \\
191.0350 \\
165.0559 \\
161.0248 \\
147.0454\end{array}$ & 432.1266 & 432.1268 & -0.4 & isoasperulosidic acid & I & $\sqrt{ }$ & $\sqrt{ }$ \\
\hline 5 & 3.67 & $\mathrm{C}_{17} \mathrm{H}_{26} \mathrm{O}_{11}$ & $451.1454[\mathrm{M}+\mathrm{HCOO}]^{-}$ & 243.0875 & 406.1472 & 406.1475 & -0.8 & harpagide acetate & $\mathrm{I}$ & $\sqrt{ }$ & $\sqrt{ }$ \\
\hline 6 & 4.10 & $\mathrm{C}_{18} \mathrm{H}_{24} \mathrm{O}_{12}$ & $431.1196[\mathrm{M}-\mathrm{H}]^{-}$ & $\begin{array}{l}371.0974 \\
251.0563 \\
165.0560\end{array}$ & 432.1269 & 432.1268 & 0.3 & asperulosidic acid & I & $\sqrt{ }$ & $\sqrt{ }$ \\
\hline 7 & 4.78 & $\mathrm{C}_{21} \mathrm{H}_{32} \mathrm{O}_{15}$ & $523.1666[\mathrm{M}-\mathrm{H}]^{-}$ & $\begin{array}{l}477.1613 \\
293.0876 \\
233.0669\end{array}$ & 524.1739 & 524.1741 & -0.4 & rehmannioside A & I & $\sqrt{ }$ & $\sqrt{ }$ \\
\hline 8 & 4.86 & $\mathrm{C}_{18} \mathrm{H}_{22} \mathrm{O}_{11}$ & $459.1143\left[\mathrm{M}+\mathrm{HCOO}^{-}\right.$ & 147.0454 & 414.1161 & 414.1162 & -0.3 & asperuloside & $\mathrm{I}$ & $\sqrt{ }$ & $\sqrt{ }$ \\
\hline 9 & 6.44 & $\mathrm{C}_{26} \mathrm{H}_{28} \mathrm{O}_{15}$ & $579.1342[\mathrm{M}-\mathrm{H}]^{-}$ & $\begin{array}{l}413.1078 \\
285.0398 \\
146.9650\end{array}$ & 580.1415 & 580.1428 & -2.4 & $\begin{array}{l}\text { 3-O-primeverose-1,6,8- } \\
\text { trihydroxy-2-methyl- } \\
\text { anthraquinone }\end{array}$ & A & & $\sqrt{ }$ \\
\hline 10 & 6.84 & $\mathrm{C}_{27} \mathrm{H}_{30} \mathrm{O}_{15}$ & $593.1507[\mathrm{M}-\mathrm{H}]^{-}$ & 253.0506 & 594.1580 & 594.1585 & -0.8 & $\begin{array}{l}\text { 1-O-primeverose-3-methoxy- } \\
\text { 8-hydroxy-2-methylol-anthraquinone }\end{array}$ & $\mathrm{A}$ & $\sqrt{ }$ & $\sqrt{ }$ \\
\hline 11 & 6.89 & $\mathrm{C}_{27} \mathrm{H}_{30} \mathrm{O}_{14}$ & $623.1616\left[\mathrm{M}+\mathrm{HCOO}^{-}\right.$ & $\begin{array}{l}283.0612 \\
253.0506 \\
146.9654\end{array}$ & 578.1634 & 578.1636 & -0.2 & $\begin{array}{l}\text { 1-O-gentiobiose-2-methylol- } \\
\text { anthraquinone }\end{array}$ & $\mathrm{A}$ & $\sqrt{ }$ & $\sqrt{ }$ \\
\hline 12 & 7.21 & $\mathrm{C}_{22} \mathrm{H}_{22} \mathrm{O}_{10}$ & $491.1195\left[\mathrm{M}+\mathrm{HCOO}^{-}\right.$ & 283.0613 & 446.1213 & 446.1213 & -0.1 & $\begin{array}{l}\text { 1-O- } \beta \text {-D-glycopyranosyl-8- } \\
\text { methoxy-emodin }\end{array}$ & A & & $\sqrt{ }$ \\
\hline 13 & 7.23 & $\mathrm{C}_{28} \mathrm{H}_{32} \mathrm{O}_{14}$ & $637.1771\left[\mathrm{M}+\mathrm{HCOO}^{-}\right.$ & $\begin{array}{l}547.1449 \\
307.0609 \\
297.0763 \\
283.0613\end{array}$ & 592.1789 & 592.1792 & -0.4 & $\begin{array}{l}\text { 1-O-primeverose-3,8-dimethoxy-2- } \\
\text { methyl-anthraquinone }\end{array}$ & A & & $\sqrt{ }$ \\
\hline
\end{tabular}


Table 1. Cont.

\begin{tabular}{|c|c|c|c|c|c|c|c|c|c|c|c|}
\hline \multirow{2}{*}{$\begin{array}{c}\text { Peak } \\
\text { No. }\end{array}$} & \multirow{2}{*}{$\mathbf{R t}^{\mathrm{e}}(\min )$} & \multirow{2}{*}{ Formula } & \multirow{2}{*}{ Quasi-Molecular Ion } & \multirow{2}{*}{ MS $^{2}$ Ions } & \multicolumn{2}{|c|}{ Molecular Weight } & \multirow{2}{*}{$\begin{array}{c}\text { Mass } \\
\text { Error (ppm) }\end{array}$} & \multirow{2}{*}{ Identification } & \multirow{2}{*}{ Type } & \multicolumn{2}{|c|}{ Source } \\
\hline & & & & & Observed & Theoretical & & & & M. O & M. C \\
\hline 14 & 7.25 & $\mathrm{C}_{27} \mathrm{H}_{30} \mathrm{O}_{15}$ & $593.1510[\mathrm{M}-\mathrm{H}]^{-}$ & $\begin{array}{l}269.0456 \\
251.0350 \\
146.9655\end{array}$ & 594.1583 & 594.1585 & -0.3 & 1-O-gentiobiose-emodin & $\mathrm{A}$ & $\sqrt{ }$ & \\
\hline 15 & 7.27 & $\mathrm{C}_{26} \mathrm{H}_{28} \mathrm{O}_{14}$ & $563.1405[\mathrm{M}-\mathrm{H}]^{-}$ & $\begin{array}{l}269.0457 \\
251.0352 \\
237.0557\end{array}$ & 564.1478 & 564.1479 & -0.2 & 1-O-primeverose-aloeemodin & $\mathrm{A}$ & $\sqrt{ }$ & $\sqrt{ }$ \\
\hline 16 & 7.34 & $\mathrm{C}_{27} \mathrm{H}_{30} \mathrm{O}_{14}$ & $577.1561[\mathrm{M}-\mathrm{H}]^{-}$ & 253.0504 & 578.1634 & 578.1636 & -0.3 & $\begin{array}{l}\text { 3-O-gentiobiose -1-hydroxy- } \\
\text { 2-methyl-anthraquinone }\end{array}$ & A & $\sqrt{ }$ & $\sqrt{ }$ \\
\hline 17 & 7.40 & $\mathrm{C}_{27} \mathrm{H}_{30} \mathrm{O}_{14}$ & 623.1617[M + $\mathrm{HCOO}^{-}$ & 269.0455 & 578.1635 & 578.1636 & -0.1 & $\begin{array}{l}\text { 8-O-primeverose-1-methoxy-3- } \\
\text { hydroxy-2- methyl } \\
\text {-anthraquinone }\end{array}$ & $\mathrm{A}$ & $\sqrt{ }$ & \\
\hline 18 & 7.53 & $\mathrm{C}_{27} \mathrm{H}_{30} \mathrm{O}_{14}$ & $577.1559[\mathrm{M}-\mathrm{H}]^{-}$ & 283.0619 & 578.1632 & 578.1636 & -0.7 & $\begin{array}{l}\text { 3-O-primeverose-8-methoxy-1- } \\
\text { hydroxy- 2- methyl } \\
\text {-anthraquinone }\end{array}$ & $\mathrm{A}$ & $\sqrt{ }$ & \\
\hline 19 & 7.57 & $\mathrm{C}_{26} \mathrm{H}_{28} \mathrm{O}_{14}$ & $609.1461\left[\mathrm{M}+\mathrm{HCOO}^{-}\right.$ & $\begin{array}{l}415.1024 \\
269.0457\end{array}$ & 564.1479 & 564.1479 & 0.0 & $\begin{array}{l}\text { 8-O-primeverose-1,3-dihydroxy-2- } \\
\text { methyl -anthraquinone }\end{array}$ & $\mathrm{A}$ & $\sqrt{ }$ & \\
\hline 20 & 7.61 & $\mathrm{C}_{27} \mathrm{H}_{30} \mathrm{O}_{13}$ & $607.1663\left[\mathrm{M}+\mathrm{HCOO}^{-}\right.$ & 253.0505 & 562.1681 & 562.1686 & -0.8 & $\begin{array}{l}\text { 1-O-gentiobiose -3-hydroxy- } \\
\text { 2-methyl -anthraquinone }\end{array}$ & $\mathrm{A}$ & $\sqrt{ }$ & $\sqrt{ }$ \\
\hline 21 & 7.65 & $\mathrm{C}_{21} \mathrm{H}_{20} \mathrm{O}_{10}$ & $431.0977[\mathrm{M}-\mathrm{H}]^{-}$ & $\begin{array}{l}269.0455 \\
251.0347\end{array}$ & 432.1049 & 432.1057 & -1.7 & emodin 1-O- $\beta$-D-glycopyranosyl & $\mathrm{A}$ & & $\sqrt{ }$ \\
\hline 22 & 7.74 & $\mathrm{C}_{26} \mathrm{H}_{28} \mathrm{O}_{14}$ & $563.1404[\mathrm{M}-\mathrm{H}]^{-}$ & $\begin{array}{l}269.0456 \\
251.0348 \\
223.0407\end{array}$ & 564.1477 & 564.1479 & -0.4 & $\begin{array}{l}\text { 1-O-primeverose-3,8-dihydroxy-2- } \\
\text { methyl -anthraquinone }\end{array}$ & $\mathrm{A}$ & $\sqrt{ }$ & $\sqrt{ }$ \\
\hline 23 & 7.75 & $\mathrm{C}_{28} \mathrm{H}_{32} \mathrm{O}_{15}$ & $607.1665[\mathrm{M}-\mathrm{H}]^{-}$ & $\begin{array}{l}563.1403 \\
283.0617 \\
267.0661 \\
251.0348\end{array}$ & 608.1738 & 608.1741 & -0.6 & 1-O-gentiobiose-8-methoxy-aloeemodin & $\mathrm{A}$ & & $\sqrt{ }$ \\
\hline 24 & 7.80 & $\mathrm{C}_{27} \mathrm{H}_{30} \mathrm{O}_{14}$ & $577.1556[\mathrm{M}-\mathrm{H}]^{-}$ & 283.0618 & 578.1629 & 578.1636 & -1.2 & $\begin{array}{l}\text { 1-O-primeverose-8-methoxy-3- } \\
\text { hydroxy-2-methyl-anthraquinone }\end{array}$ & $\mathrm{A}$ & $\sqrt{ }$ & \\
\hline 25 & 7.87 & $\mathrm{C}_{27} \mathrm{H}_{30} \mathrm{O}_{15}$ & $639.1558\left[\mathrm{M}+\mathrm{HCOO}^{-}\right.$ & 299.0562 & 594.1576 & 594.1585 & -1.3 & $\begin{array}{l}\text { 1-O-primeverose-3,4-dimethoxy-2- } \\
\text { hydroxy-anthraquinone }\end{array}$ & $\mathrm{A}$ & $\sqrt{ }$ & \\
\hline 26 & 7.89 & $\mathrm{C}_{26} \mathrm{H}_{28} \mathrm{O}_{14}$ & $563.1405[\mathrm{M}-\mathrm{H}]^{-}$ & 269.0457 & 564.1478 & 564.1479 & -0.2 & $\begin{array}{l}\text { 3-O-primeverose-1,8-dihydroxy-2- } \\
\text { methyl -anthraquinone }\end{array}$ & $\mathrm{A}$ & $\sqrt{ }$ & \\
\hline 27 & 7.93 & $\mathrm{C}_{27} \mathrm{H}_{30} \mathrm{O}_{15}$ & $593.1502[\mathrm{M}-\mathrm{H}]^{-}$ & $\begin{array}{l}547.1452 \\
283.0608 \\
285.0405 \\
251.0347\end{array}$ & 594.1574 & 594.1585 & -1.7 & $\begin{array}{l}\text { 1-O-primeverose-2- } \\
\text { methylol-3-hydroxy-8- } \\
\text { methoxy-anthraquinone }\end{array}$ & $\mathrm{A}$ & & $\sqrt{ }$ \\
\hline
\end{tabular}


Table 1. Cont

\begin{tabular}{|c|c|c|c|c|c|c|c|c|c|c|c|}
\hline \multirow{2}{*}{$\begin{array}{l}\text { Peak } \\
\text { No. }\end{array}$} & \multirow{2}{*}{$\operatorname{Rt}^{\mathrm{e}}(\min )$} & \multirow{2}{*}{ Formula } & \multirow{2}{*}{ Quasi-Molecular Ion } & \multirow{2}{*}{ MS $^{2}$ Ions } & \multicolumn{2}{|c|}{ Molecular Weight } & \multirow{2}{*}{$\begin{array}{c}\text { Mass } \\
\text { Error (ppm) }\end{array}$} & \multirow{2}{*}{ Identification } & \multirow{2}{*}{ Type } & \multicolumn{2}{|c|}{ Source } \\
\hline & & & & & Observed & Theoretical & & & & M. O & M. C \\
\hline 28 & 8.08 & $\mathrm{C}_{22} \mathrm{H}_{22} \mathrm{O}_{9}$ & $475.1243\left[\mathrm{M}+\mathrm{HCOO}^{-}\right.$ & $\begin{array}{l}267.0658 \\
253.0506\end{array}$ & 430.1261 & 430.1264 & -0.5 & $\begin{array}{l}\text { 1-O- } \beta \text {-D-glycopyranosylrubiadin3- } \\
\text { methyl ether }\end{array}$ & $\mathrm{A}$ & & $\sqrt{ }$ \\
\hline 29 & 8.10 & $\mathrm{C}_{26} \mathrm{H}_{28} \mathrm{O}_{13}$ & $547.1451[\mathrm{M}-\mathrm{H}]^{-}$ & 253.0506 & 548.1524 & 548.1530 & -1.1 & $\begin{array}{l}\text { 1-O-primeverose-2- } \\
\text { methylol-anthraquinone }\end{array}$ & A & & $\sqrt{ }$ \\
\hline 30 & 8.15 & $\mathrm{C}_{21} \mathrm{H}_{20} \mathrm{O}_{10}$ & $431.0977[\mathrm{M}-\mathrm{H}]^{-}$ & 269.0456 & 432.1050 & 432.1057 & -1.5 & 1-O- $\beta$-D-glycopyranosyl-emodin & A & & $\sqrt{ }$ \\
\hline 31 & 8.25 & $\mathrm{C}_{27} \mathrm{H}_{30} \mathrm{O}_{15}$ & $593.1514[\mathrm{M}-\mathrm{H}]^{-}$ & $\begin{array}{l}269.0457 \\
265.0501\end{array}$ & 594.1587 & 594.1585 & 0.4 & 1-O-gentiobiose-emodin & $\mathrm{A}$ & $\sqrt{ }$ & $\sqrt{ }$ \\
\hline 32 & 8.37 & $\mathrm{C}_{28} \mathrm{H}_{32} \mathrm{O}_{15}$ & $653.1720\left[\mathrm{M}+\mathrm{HCOO}^{-}\right.$ & $\begin{array}{l}313.0717 \\
298.0478\end{array}$ & 608.1738 & 608.1741 & -0.6 & 1-O-primeverose-8-hydroxy-ibericin & A & & $\sqrt{ }$ \\
\hline 33 & 8.40 & $\mathrm{C}_{27} \mathrm{H}_{30} \mathrm{O}_{15}$ & $593.1512[\mathrm{M}-\mathrm{H}]^{-}$ & $\begin{array}{l}547.1455 \\
431.0981 \\
269.0455 \\
\end{array}$ & 594.1585 & 594.1585 & 0.1 & 8-O-gentiobiose-emodin & A & $\sqrt{ }$ & $\sqrt{ }$ \\
\hline 34 & 8.59 & $\mathrm{C}_{26} \mathrm{H}_{28} \mathrm{O}_{13}$ & $593.1513\left[\mathrm{M}+\mathrm{HCOO}^{-}\right.$ & $\begin{array}{l}253.0508 \\
146.9655\end{array}$ & 548.1531 & 548.1530 & 0.2 & 1-O-primeverose-rubiadin & $\mathrm{A}$ & $\sqrt{ }$ & $\sqrt{ }$ \\
\hline 35 & 8.67 & $\mathrm{C}_{26} \mathrm{H}_{28} \mathrm{O}_{14}$ & $563.1407[\mathrm{M}-\mathrm{H}]^{-}$ & - & 564.1479 & 564.1479 & 0.0 & 1-O-primeverose-emodin & $\mathrm{A}$ & $\sqrt{ }$ & $\sqrt{ }$ \\
\hline 36 & 8.81 & $\mathrm{C}_{26} \mathrm{H}_{28} \mathrm{O}_{15}$ & $579.1353[\mathrm{M}-\mathrm{H}]^{-}$ & 285.0403 & 580.1426 & 580.1428 & -0.4 & $\begin{array}{l}\text { 1-O-primeverose-2-methyl- } \\
\text { 3,6,8-trihydroxy-anthraquinone }\end{array}$ & $\mathrm{A}$ & & $\sqrt{ }$ \\
\hline 37 & 8.84 & $\mathrm{C}_{30} \mathrm{H}_{48} \mathrm{O}_{7}$ & $519.3323[\mathrm{M}-\mathrm{H}]^{-}$ & 489.3215 & 520.3396 & 520.3400 & -0.8 & $\begin{array}{l}(3 \alpha)-3,19,20,24,30-p e n t a h y d r o x y u r s- \\
\text { 12-en-28-oic acid }\end{array}$ & $\mathrm{T}$ & $\sqrt{ }$ & \\
\hline 38 & 8.98 & $\mathrm{C}_{15} \mathrm{H}_{10} \mathrm{O}_{4}$ & $253.0509[\mathrm{M}-\mathrm{H}]^{-}$ & 223.0402 & 254.0582 & 254.0579 & 1.1 & 1-hydroxy-2-methylol-anthraquinone & A & $\sqrt{ }$ & $\sqrt{ }$ \\
\hline 39 & 9.00 & $\mathrm{C}_{15} \mathrm{H}_{10} \mathrm{O}_{5}$ & $269.0457[\mathrm{M}-\mathrm{H}]^{-}$ & - & 270.0530 & 270.0528 & 0.5 & aloe-emodin & $\mathrm{A}$ & & $\sqrt{ }$ \\
\hline 40 & 9.07 & $\mathrm{C}_{15} \mathrm{H}_{10} \mathrm{O}_{3}$ & $283.0614\left[\mathrm{M}+\mathrm{HCOO}^{-}\right.$ & - & 238.0632 & 238.0630 & 0.9 & 1-methyl-3-hydroxy-anthraquinone & $\mathrm{A}$ & $\sqrt{ }$ & $\sqrt{ }$ \\
\hline 41 & 9.08 & $\mathrm{C}_{15} \mathrm{H}_{10} \mathrm{O}_{4}$ & $253.0510[\mathrm{M}-\mathrm{H}]^{-}$ & - & 254.0582 & 254.0579 & 1.3 & rubiadin & $\mathrm{A}$ & $\sqrt{ }$ & $\sqrt{ }$ \\
\hline 42 & 9.79 & $\mathrm{C}_{19} \mathrm{H}_{16} \mathrm{O}_{7}$ & $401.0877\left[^{\mathrm{M}}+\mathrm{HCOO}^{-}\right.$ & $\begin{array}{l}283.0607 \\
269.0452\end{array}$ & 356.0895 & 356.0896 & -0.3 & fridamycin E & A & $\sqrt{ }$ & $\sqrt{ }$ \\
\hline 43 & 10.07 & $\mathrm{C}_{15} \mathrm{H}_{10} \mathrm{O}_{5}$ & $269.0458[\mathrm{M}-\mathrm{H}]^{-}$ & 251.0353 & 270.0531 & 270.0528 & 0.9 & emodin & $\mathrm{A}$ & $\sqrt{ }$ & $\sqrt{ }$ \\
\hline 44 & 10.27 & $\mathrm{C}_{15} \mathrm{H}_{10} \mathrm{O}_{4}$ & $253.0511[\mathrm{M}-\mathrm{H}]^{-}$ & 238.0275 & 254.0584 & 254.0579 & 1.8 & 3-hydroxy-2-methylol-anthraquinone & A & $\sqrt{ }$ & \\
\hline 45 & 10.46 & $\mathrm{C}_{30} \mathrm{H}_{46} \mathrm{O}_{6}$ & $501.3222[\mathrm{M}-\mathrm{H}]^{-}$ & $\begin{array}{l}483.3111 \\
457.3323\end{array}$ & 502.3295 & 502.3294 & 0.1 & $\begin{array}{l}(3 \alpha, 19 \beta)-3,19,23-\text { trihydroxy-6- } \\
\text { oxoolean-12-en-28-oic acid }\end{array}$ & $\mathrm{T}$ & $\sqrt{ }$ & \\
\hline 46 & 10.53 & $\mathrm{C}_{15} \mathrm{H}_{10} \mathrm{O}_{3}$ & $283.0614\left[\mathrm{M}+\mathrm{HCOO}^{-}\right.$ & - & 238.0632 & 238.0630 & 0.7 & 1-hydroxy-2-methyl-anthraquinone & A & & $\sqrt{ }$ \\
\hline 47 & 10.82 & $\mathrm{C}_{15} \mathrm{H}_{10} \mathrm{O}_{5}$ & $269.0457[\mathrm{M}-\mathrm{H}]^{-}$ & - & 270.0530 & 270.0528 & 0.6 & 1,3-dihydroxy-2-methoxy-anthraquinone & ne A & & $\sqrt{ }$ \\
\hline 48 & 10.97 & $\mathrm{C}_{30} \mathrm{H}_{48} \mathrm{O}_{6}$ & $549.3426\left[\mathrm{M}+\mathrm{HCOO}^{-}\right.$ & 485.3259 & 504.3444 & 504.3451 & -1.2 & $\begin{array}{l}\text { 2,3,6,23-tetrahydroxyurs-12-en-28-oic } \\
\text { acid }\end{array}$ & $\mathrm{T}$ & $\sqrt{ }$ & \\
\hline
\end{tabular}


Table 1. Cont.

\begin{tabular}{|c|c|c|c|c|c|c|c|c|c|c|c|}
\hline \multirow{2}{*}{$\begin{array}{c}\text { Peak } \\
\text { No. }\end{array}$} & \multirow{2}{*}{$\mathrm{Rt}^{\mathrm{e}}(\min )$} & \multirow{2}{*}{ Formula } & \multirow{2}{*}{ Quasi-Molecular Ion } & \multirow{2}{*}{ MS $^{2}$ Ions } & \multicolumn{2}{|c|}{ Molecular Weight } & \multirow{2}{*}{$\begin{array}{c}\text { Mass } \\
\text { Error (ppm) }\end{array}$} & \multirow{2}{*}{ Identification } & \multirow{2}{*}{ Type } & \multicolumn{2}{|c|}{ Source } \\
\hline & & & & & Observed & Theoretical & & & & M. $\mathrm{O}$ & M. C \\
\hline 49 & 11.00 & $\mathrm{C}_{16} \mathrm{H}_{12} \mathrm{O}_{4}$ & $267.0658[\mathrm{M}-\mathrm{H}]^{-}$ & $\begin{array}{l}252.0424 \\
224.0470\end{array}$ & 268.0731 & 268.0736 & -1.7 & rubiadin 3-methyl ether & A & $\sqrt{ }$ & \\
\hline 50 & 11.06 & $\mathrm{C}_{30} \mathrm{H}_{48} \mathrm{O}_{6}$ & $503.3377[\mathrm{M}-\mathrm{H}]^{-}$ & 485.3268 & 504.3449 & 504.3451 & -0.3 & $\begin{array}{l}\text { 2,3,19,23-tetrahydroxyolean-12- } \\
\text { en-28-oic acid }\end{array}$ & $\mathrm{T}$ & $\sqrt{ }$ & \\
\hline 51 & 11.14 & $\mathrm{C}_{16} \mathrm{H}_{12} \mathrm{O}_{4}$ & $267.0665[\mathrm{M}-\mathrm{H}]^{-}$ & 224.0476 & 268.0738 & 268.0736 & 0.7 & rubiadin 1-methyl ether & A & $\sqrt{ }$ & $\sqrt{ }$ \\
\hline 52 & 11.97 & $\mathrm{C}_{30} \mathrm{H}_{48} \mathrm{O}_{5}$ & $487.3424[\mathrm{M}-\mathrm{H}]^{-}$ & $\begin{array}{l}469.3322 \\
437.3048\end{array}$ & 488.3497 & 488.3502 & -1.0 & $\begin{array}{l}(3 \beta, 6 \alpha)-3,6,24 \text {-trihydroxyolean-12- } \\
\text { en-28-oic acid }\end{array}$ & $\mathrm{T}$ & $\sqrt{ }$ & \\
\hline 53 & 12.26 & $\mathrm{C}_{30} \mathrm{H}_{48} \mathrm{O}_{5}$ & $485.3273[\mathrm{M}-\mathrm{H}]^{-}$ & $\begin{array}{l}467.3164 \\
441.3372 \\
423.3266\end{array}$ & 486.3346 & 486.3345 & 0.2 & $\begin{array}{l}(3 \beta, 6 \alpha)-3,6,23 \text {-trihydroxyursa- } \\
\text { 12,19(29)-dien-28-oic acid }\end{array}$ & $\mathrm{T}$ & $\sqrt{ }$ & \\
\hline 54 & 12.91 & $\mathrm{C}_{30} \mathrm{H}_{48} \mathrm{O}_{5}$ & $487.3433[\mathrm{M}-\mathrm{H}]^{-}$ & $\begin{array}{l}469.3324 \\
443.3528 \\
425.3422\end{array}$ & 488.3506 & 488.3502 & 0.9 & $\begin{array}{c}(2 \alpha, 3 \beta, 19 \alpha)-2,3,19 \text {-trihydroxyolean- } \\
12 \text {-en-28-oic acid }\end{array}$ & $\mathrm{T}$ & $\sqrt{ }$ & \\
\hline 55 & 13.01 & $\mathrm{C}_{30} \mathrm{H}_{48} \mathrm{O}_{5}$ & $487.3429[\mathrm{M}-\mathrm{H}]^{-}$ & $\begin{array}{l}441.3368 \\
425.3424 \\
409.3109\end{array}$ & 488.3501 & 488.3502 & -0.1 & $\begin{array}{c}(2 \alpha, 3 \alpha)-2,3,24 \text {-trihydroxyolean- } \\
18 \text {-en-28-oic acid }\end{array}$ & $\mathrm{T}$ & $\sqrt{ }$ & \\
\hline 56 & 13.06 & $\mathrm{C}_{17} \mathrm{H}_{14} \mathrm{O}_{5}$ & $297.0766[\mathrm{M}-\mathrm{H}]^{-}$ & 251.0348 & 298.0839 & 298.08412 & -0.8 & ibericin & A & $\sqrt{ }$ & \\
\hline 57 & 13.11 & $\mathrm{C}_{30} \mathrm{H}_{48} \mathrm{O}_{5}$ & $487.3425[\mathrm{M}-\mathrm{H}]^{-}$ & $\begin{array}{l}441.3368 \\
425.3424 \\
409.3109 \\
\end{array}$ & 488.3498 & 488.3502 & -0.8 & 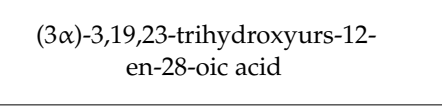 & $\mathrm{T}$ & $\sqrt{ }$ & \\
\hline 58 & 13.26 & $\mathrm{C}_{30} \mathrm{H}_{48} \mathrm{O}_{5}$ & $533.3475\left[^{\mathrm{M}+\mathrm{HCOO}^{-}}\right.$ & 441.3364 & 488.3493 & 488.3502 & -1.7 & $\begin{array}{l}\text { 2,3,23-trihydroxyurs-12-en-28-oic } \\
\text { acid }\end{array}$ & $\mathrm{T}$ & $\sqrt{ }$ & \\
\hline 59 & 13.53 & $\mathrm{C}_{33} \mathrm{H}_{50} \mathrm{O}_{8}$ & $573.3427[\mathrm{M}-\mathrm{H}]^{-}$ & - & 574.3499 & 574.3506 & -1.1 & $\begin{array}{c}\text { butyl }(3 \beta, 7 \beta, 12 \beta)-12- \\
\text { acetoxy-3,7-dihydroxy- } \\
\text { 4,4,14-trimethyl-11,15-dioxochol- } \\
\text { 8-en-24-oate }\end{array}$ & $\mathrm{T}$ & $\sqrt{ }$ & \\
\hline 60 & 14.30 & $\mathrm{C}_{31} \mathrm{H}_{50} \mathrm{O}_{6}$ & $517.3527[\mathrm{M}-\mathrm{H}]^{-}$ & 471.3470 & 518.3600 & 518.3607 & -1.4 & $\begin{array}{c}(1 \alpha, 3 \beta, 9 \beta) \text {-24-hydroperoxy-1,3- } \\
\text { dihydroxy-5-methyl-9,19-cyclolanost- } \\
25 \text {-en-28-oic acid }\end{array}$ & $\mathrm{T}$ & $\sqrt{ }$ & \\
\hline 61 & 14.61 & $\mathrm{C}_{30} \mathrm{H}_{48} \mathrm{O}_{4}$ & $471.3474[\mathrm{M}-\mathrm{H}]^{-}$ & $\begin{array}{l}453.3369 \\
427.3583 \\
\end{array}$ & 472.3547 & 472.3553 & -1.2 & hederagenin & $\mathrm{T}$ & $\sqrt{ }$ & \\
\hline 62 & 15.95 & $\mathrm{C}_{30} \mathrm{H}_{48} \mathrm{O}_{4}$ & $517.3528\left[^{\mathrm{M}}+\mathrm{HCOO}^{-}\right.$ & 423.3266 & 472.3546 & 472.3553 & -1.3 & maslinic acid & $\mathrm{T}$ & $\sqrt{ }$ & \\
\hline 63 & 19.52 & $\mathrm{C}_{29} \mathrm{H}_{44} \mathrm{O}_{2}$ & $423.3264[\mathrm{M}-\mathrm{H}]^{-}$ & 325.0718 & 424.3337 & 424.3341 & -1.1 & camphoratin $\mathrm{H}$ & $\mathrm{T}$ & $\sqrt{ }$ & $\sqrt{ }$ \\
\hline
\end{tabular}

I: iridoid glycosides; A: anthraquinones; T: triterpenes. M. O: M. officinalis; M. C: M. citrifolia. 

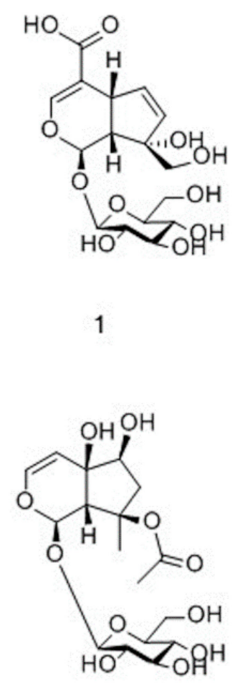

5
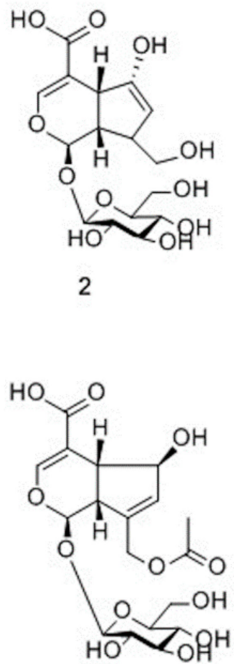

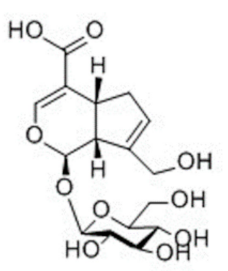

3

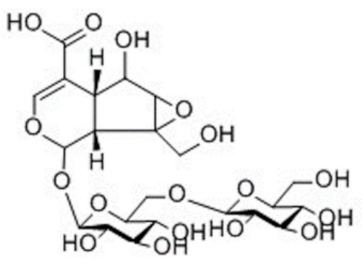

7

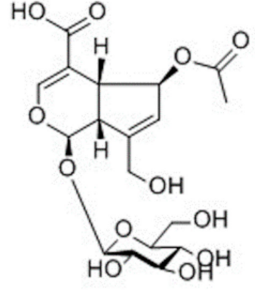

4

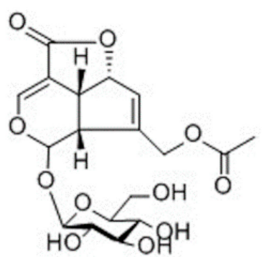

Figure 2. Chemical structures of iridoid glycosides identified in M. citrifolia and M. officinalis.

Compounds 9-36, 38-44, 46-47, 49, 51, 56 were characterized as anthraquinones, and their structures are displayed in Figure 3. Based on whether they had a glycosyl group, the compounds were further classified into either anthraquinone glycosides $(9-36,41)$ or anthraquinone aglycones (38-40, 42-44, 46-47, 49, 51, 56). The parent ions of anthraquinone glycosides yielded fragment ions by effortlessly losing a glucose unit (162), xyloside unit (132), $\mathrm{CH}_{2}$ (14), and $\mathrm{HCOOH}$ (46) [17-19]. The proposed fragmentation pathway is shown in Figure S2. By contrast, anthraquinone aglycones were relatively stable, thus their fragments were difficult to determine.

Compounds 37, 45, 48, 50, 52-55, 57-63 were determined as triterpenes, and their structures are displayed in Figure 4 [20]. The structure of triterpenes is relatively stable, and it commonly formed ions that were produced by the loss of a unit of $\mathrm{H}_{2} \mathrm{O}$. To date, approximately 100 compounds have been isolated from $M$. officinalis, which mainly are polysaccharides, oligosaccharides, anthraquinones, triterpenes, and iridoid glycosides [2]. Approximately, 160 compounds have been isolated and identified from $M$. citrifolia, which mainly comprise anthraquinones, organic acids, alkaloid, terpenes and volatile compounds [11]. In the present study, 49 compounds from the methanol extract of M. officinalis roots were identified, classified into 26 anthraquinones, 15 triterpenes, and 8 iridoid glycosides. At the same time, 39 compounds were identified from the methanol extract of $M$. citrifolia roots, including 30 anthraquinones, 1 triterpene, and 8 iridoid glycosides. Among them, 25 compounds were found to be present in both plants. These compounds are classified into 16 anthraquinones, 1 triterpene, and 8 iridoid glycosides. Detailed information on the identified compounds is shown in Table 1. 
<smiles>[R2]Cc1c([R3])cc2c(c1Br)C(=O)c1ccccc1C2=O</smiles>

$11 R_{1}=$ O-gent, $R_{2}=O H, R_{3}=H$ $16 R_{1}=O H, R_{2}=H, R_{2}=O$-gent $20 R_{1}=$ O-gent, $R_{2}=H_{1} R_{3}=O H$ $28 R_{1}=O$-glu, $R_{2}=H_{1} R_{3}=O_{3}$ $29 \mathrm{R}_{1}=\mathrm{O}$-prim, $\mathrm{R}_{2}=\mathrm{OH}, \mathrm{R}_{3}=\mathrm{H}$ $34 \mathrm{R}_{1}=\mathrm{O}$-prim, $\mathrm{R}_{2}=\mathrm{H}, \mathrm{R}_{3}=\mathrm{OH}$ $38 \mathrm{R}_{1}=\mathrm{H}, \mathrm{R}_{2}=\mathrm{OH}, \mathrm{R}_{3}=\mathrm{H}$

$40 \mathrm{R}_{1}=\mathrm{CH}_{3}, \mathrm{R}_{2}=\mathrm{H}, \mathrm{R}_{3}=\mathrm{OH}$

$44 \mathrm{R}_{1}=\mathrm{H}, \mathrm{R}_{2}=\mathrm{OH}, \mathrm{R}_{3}=\mathrm{OH}$

$46 \mathrm{R}_{1}=\mathrm{OH}, \mathrm{R}_{2}=\mathrm{H}, \mathrm{R}_{3}=\mathrm{H}$

$49 \mathrm{R}_{1}=\mathrm{OH}, \mathrm{R}_{2}=\mathrm{H}, \mathrm{R}_{3}=\mathrm{OCH}_{3}$

$51 \mathrm{R}_{1}=\mathrm{OCH}_{3}, \mathrm{R}_{2}=\mathrm{H}, \mathrm{R}_{3}=\mathrm{OH}$

$56 \mathrm{R}_{1}=\mathrm{OH}, \mathrm{R}_{2}=\mathrm{OCH}_{2} \mathrm{CH}_{3}, \mathrm{R}_{3}=\mathrm{OH}$<smiles>[R6]Oc1cc([R3])cc2c1C(=O)c1cc([R20])cc([R])c1C2=O</smiles>

$12 \mathrm{R}_{1}=\mathrm{O}$-glu, $\mathrm{R}_{2}=\mathrm{CH}_{2} \mathrm{OH}, \mathrm{R}_{3}=\mathrm{OH}, \mathrm{R}_{4}=\mathrm{CH}_{3}$ $14 \mathrm{R}_{1}=\mathrm{O}$-gent, $\mathrm{R}_{2}=\mathrm{OH}, \mathrm{R}_{3}=\mathrm{CH}_{3}, \mathrm{R}_{4}=\mathrm{H}$

$15 \mathrm{R}_{1}=\mathrm{O}$-prime, $\mathrm{R}_{2}=\mathrm{CH}_{2} \mathrm{OH}, \mathrm{R}_{3}=\mathrm{H}, \mathrm{R}_{4}=\mathrm{H}$

$21 \mathrm{R}_{1}=\mathrm{OH}, \mathrm{R}_{2}=\mathrm{CH}_{3}, \mathrm{R}_{3}=\mathrm{OH}, \mathrm{R}_{4}=\mathrm{glu}$ $23 \mathrm{R}_{1}=$ O-gent, $\mathrm{R}_{2}=\mathrm{CH}_{2} \mathrm{OH}, \mathrm{R}_{3}=\mathrm{H}, \mathrm{R}_{4}=\mathrm{CH}_{3}$ $30 \mathrm{R}_{1}=\mathrm{O}$-glu, $\mathrm{R}_{2}=\mathrm{CH}_{3}, \mathrm{R}_{3}=\mathrm{OH}, \mathrm{R}_{4}=\mathrm{H}$ $31 R_{1}=$ O-gent, $R_{2}=C_{3}, R_{3}=O H, R_{4}=H$ $33 \mathrm{R}_{1}=\mathrm{OH}, \mathrm{R}_{2}=\mathrm{CH}_{3}, \mathrm{R}_{3}=\mathrm{OH}, \mathrm{R}_{4}=$ gent $35 \mathrm{R}_{1}=\mathrm{O}$-prime, $\mathrm{R}_{2}=\mathrm{CH}_{3}, \mathrm{R}_{3}=\mathrm{OH}, \mathrm{R}_{4}=\mathrm{H}$ $39 \mathrm{R}_{1}=\mathrm{OH}, \mathrm{R}_{2}=\mathrm{CH}_{2} \mathrm{OH}, \mathrm{R}_{3}=\mathrm{H}, \mathrm{R}_{4}=\mathrm{H}$ $43 \mathrm{R}_{1}=\mathrm{OH}, \mathrm{R}_{2}=\mathrm{CH}_{3}, \mathrm{R}_{3}=\mathrm{OH}, \mathrm{R}_{4}=\mathrm{H}$<smiles>COc1c(O)c(OC)c2c(c1OC)C(=O)c1ccccc1C2=O</smiles>

25<smiles>Cc1cc(O)cc2c1C(=O)c1ccccc1C2=O</smiles>

40<smiles>[R2]Cc1c(O[R6])cc2c(c1[R])C(=O)c1c(O[R20])cccc1C2=O</smiles>

$10 \mathrm{R}_{1}=\mathrm{O}$-prime, $\mathrm{R}_{2}=\mathrm{OH}, \mathrm{R}_{3}=\mathrm{CH}_{3}, \mathrm{R}_{4}=\mathrm{H}$ $13 \mathrm{R}_{1}=$ O-prime, $\mathrm{R}_{2}=\mathrm{H}, \mathrm{R}_{3}=\mathrm{CH}_{3}, \mathrm{R}_{4}=\mathrm{CH}_{3}$ $17 R_{1}=O H_{3}, R_{2}=H, R_{3}=H, R_{4}=$ prime

$18 \mathrm{R}_{1}=\mathrm{OH}, \mathrm{R}_{2}=\mathrm{H}, \mathrm{R}_{3}=$ prime, $\mathrm{R}_{4}=\mathrm{OCH}_{3}$

$19 \mathrm{R}_{1}=\mathrm{OH}, \mathrm{R}_{2}=\mathrm{H}, \mathrm{R}_{3}=\mathrm{H}, \mathrm{R}_{4}=$ prime

$22 R_{1}=$ O-prime, $R_{2}=H, R_{3}=H, R_{4}=H$

$24 R_{1}=$ O-prime, $R_{2}=H, R_{3}=H, R_{4}=C_{3}$

$26 \mathrm{R}_{1}=\mathrm{OH}, \mathrm{R}_{2}=\mathrm{H}, \mathrm{R}_{3}=$ prime, $\mathrm{R}_{4}=\mathrm{H}$

$27 \mathrm{R}_{1}=$ O-prime, $\mathrm{R}_{2}=\mathrm{OH}, \mathrm{R}_{3}=\mathrm{H}, \mathrm{R}_{4}=\mathrm{CH}_{3}$

$32 R_{1}=O$-prime, $R_{2}=O C_{2} C_{3}, R_{3}=H, R_{4}=H$<smiles>CCOc1cc2c(c(OCC)c1C)C(=O)c1c(O)cc(O)cc1C2=O</smiles>

$9 R_{1}=H_{1} R_{2}=$ prime $36 \mathrm{R}_{1}=$ prime, $\mathrm{R}_{2}=\mathrm{H}$

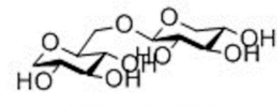

primeverose

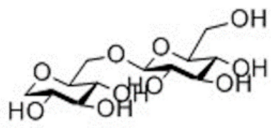

gentiobiose

Figure 3. Chemical structures of anthraquinones identified in M. citrifolia and M. officinalis. 


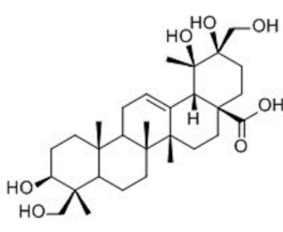

37

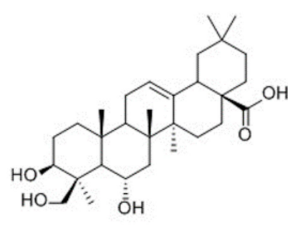

52

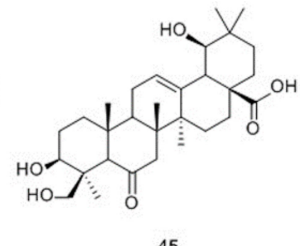

45

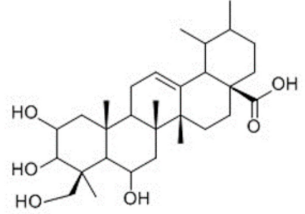

48

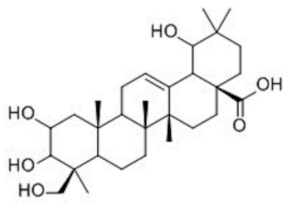

50

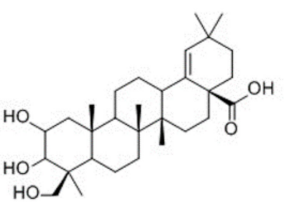

55

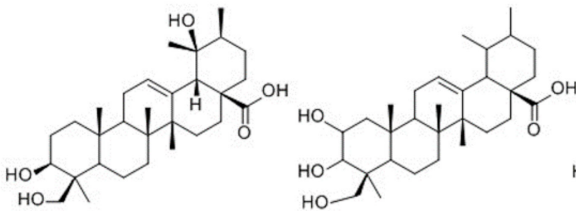

57

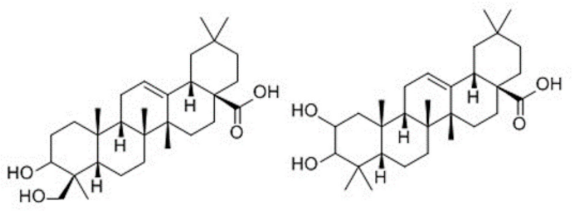

61

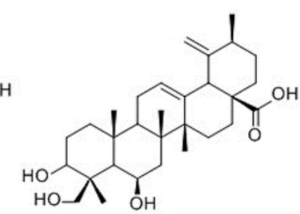

53

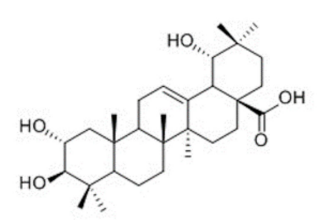

54

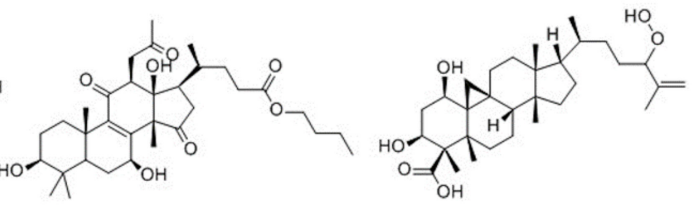

59

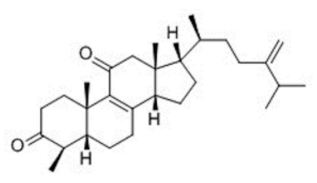

63

Figure 4. Chemical structures of triterpenoids identified in M. citrifolia and M. officinalis.

\subsection{Tentative Identification of Potential Marker Compounds from M. officinalis and M. citrifolia}

To clarify the compound differences between the methanol extracts of M. officinalis and M. citrifolia roots, an untargeted metabolite profiling of the extracts was conducted and analyzed by UPLC-QTOF-MS/MS and Progenesis QI software. The PCA scores plot (Figure 5A) indicates that the chemical compositions of $M$. officinalis and $M$. citrifolia differ from one another. M. officinalis and $M$. citrifolia root samples were clearly distinguished by the principal component 1 (PC1). Furthermore, to explore the potential biomarkers corresponding to the differences between $M$. officinalis and M. citrifolia root extracts, UPLC-QTOF-MS/MS data were processed by a supervised OPLS-DA. Through an S-plot analysis at each point, the $\mathrm{X}$ - and $Y$-axis showed the variable contributions and sample correlations. The compounds with significant differences between groups were called marker compounds, and were plotted at the top right (1) and bottom left (-1). Therefore, nine ions (red box mark) were selected as candidates, to chemically distinguish $M$. officinalis from $M$. citrifolia root extracts (Figure 5B). 
A

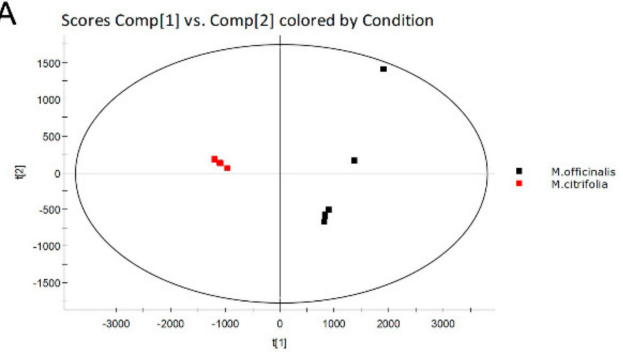

C

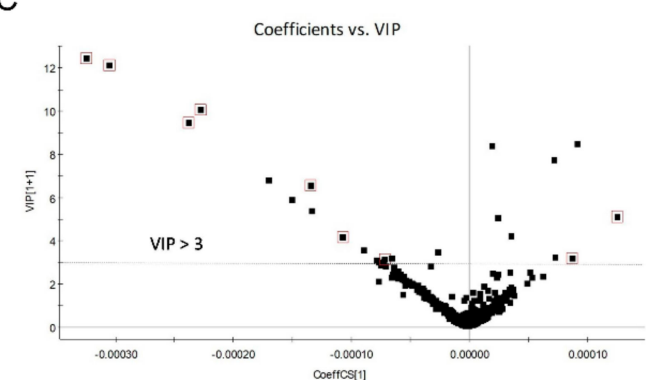

B

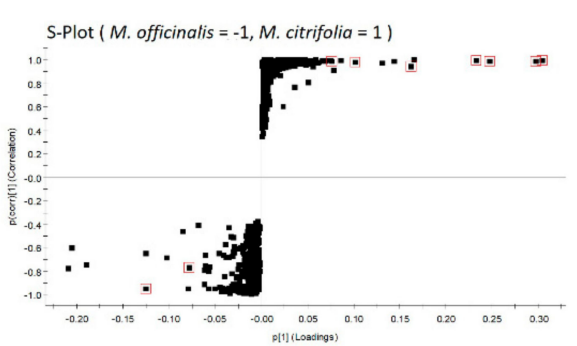

D

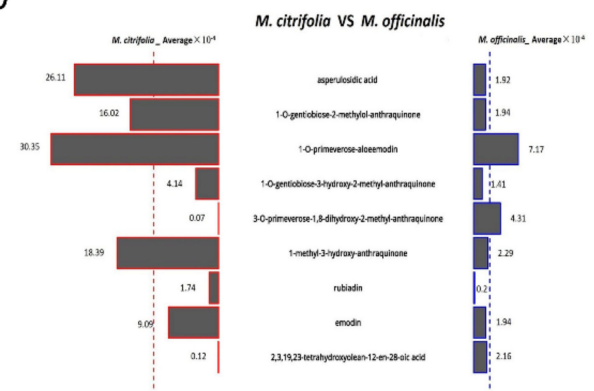

Figure 5. Selected potential biomarkers from M. officinalis and M. citrifolia based on principal component analysis (PCA) and orthogonal partial least squares-discriminant analysis (OPLS-DA). (A) PCA score plot; (B) S-Plot from OPLS-DA of M. officinalis and M. citrifolia. (C) The variable importance in projection (VIP) score of selected markers; (D) Butterfly shows the variable averages by group of selected potential marker compounds. The most differential compounds are marked with a red square.

The variable importance in projection (VIP) plot (Figure 5C) showed that all the 7 selected potential marker compounds in $M$. citrifolia root extracts and 2 selected potential marker compounds in $M$. officinalis root extract have a high VIP value (VIP > 3), suggesting that these marker compounds are largely responsible for the chemical difference between these two samples. Furthermore, the variable average between groups clearly showed content discrepancy of the selected marker compounds in M. citrifolia and M. officinalis root extracts (Figure 5D). The 9 selected markers were asperulosidic acid (6), 1-O-gentiobiose-2-methylol-anthraquinone (11), 1-O-primeverose-aloe-emodin (15), 1-O-gentiobiose-3-hydroxy-2-methyl- anthraquinone (20), 3-O-primeverose-1,8-dihydroxy-2-methyl-anthraquinone (26), 1-methyl-3-hydroxy-anthraquinone (40), rubiadin (41), emodin (43), and 2,3,19,23-tetrahydroxyolean-12-en-28-oic acid (50). The results showed that the levels of 3-O-primeverose-1,8-dihydroxy-2-methyl-anthraquinone (26) and 2,3,19,23-tetrahydroxyolean-12-en-28-oic acid (50) were relatively higher in the root extract of M. officinalis, than in M. citrifolia (Figure 5D). The other seven compounds were relatively higher in $M$. citrifolia roots. Among them, compounds 11, 15, and 40 are anthraquinones, which are the characteristic compounds of the family Rubiaceae. Asperulosidic acid (6, iridoid glucosides) is one of the most common pharmacologically active ingredients in M. officinalis [2]. Previous reports found that it exhibited anti-inflammatory, anti-renal fibrosis, and antibacterial activities [21,22].

\subsection{The Cytotoxicity of the Methanol Extracts of M. officinalis and M. citrifolia Roots}

To further investigate the differences in the methanol extracts of M. officinalis and M. citrifolia roots in terms of cytotoxicity, a CCK-8 assay was used to examine the cell viability of three liver cell lines (normal cells: LO2; cancer cells: HepG2, SMMCH771) after treatment by M. officinalis and $M$. citrifolia root extracts. As shown in Figure 6A, the two extracts were non-cytotoxic to the cells after a treatment of $24 \mathrm{~h}$ at concentrations of up to $100 \mu \mathrm{g} / \mathrm{mL}$. When treated for 48 and $72 \mathrm{~h}$, lower cell viability was observed in $M$. citrifolia root extract treatment groups compared to $M$. officinalis root extract treatment groups. In addition, a colony formation assay was used to evaluate the long-term impact of the M. officinalis and M. citrifolia root extracts on the cells. The results showed that a high dose of 
M. officinalis and M. citrifolia root extracts promoted the cell proliferation of LO2 cells, and inhibited the cell proliferation of HepG2 and SMMCH771 cells (Figure 6B). No significant differences were found between the root extracts of $M$. officinalis and $M$. citrifolia in terms of long-term impact on HepG2 and SMMCH771 cells. Hence, it is suggested that the methanol extracts of $M$. officinalis and M. citrifolia roots showed no cell cytotoxicity to normal cells, and even promoted the proliferation of normal liver cells. Meanwhile, compared to $M$. officinalis root extract, $M$. citrifolia root extracts exhibited stronger cell cytotoxicity to liver cancer cells.
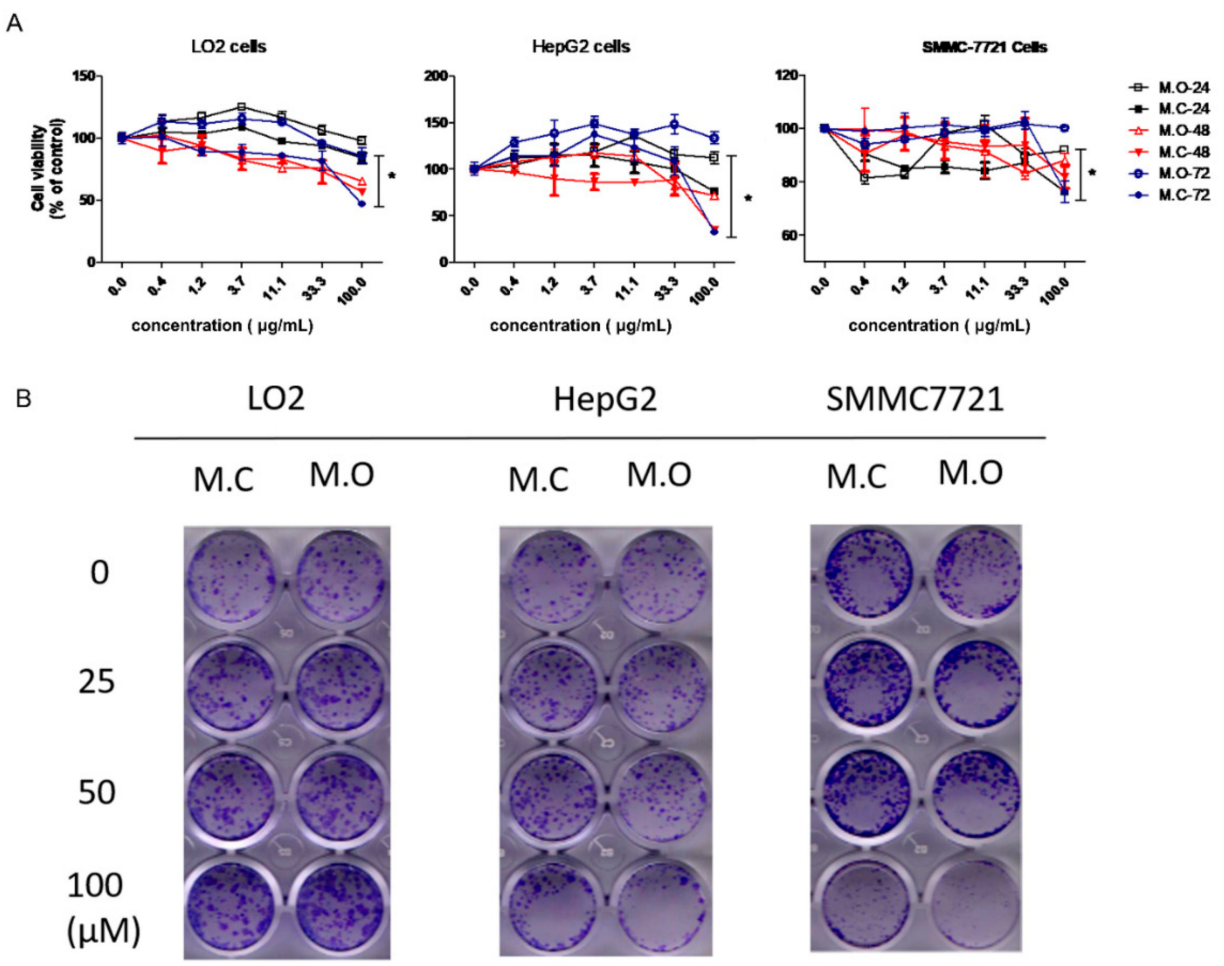

HepG2 SMMC7721
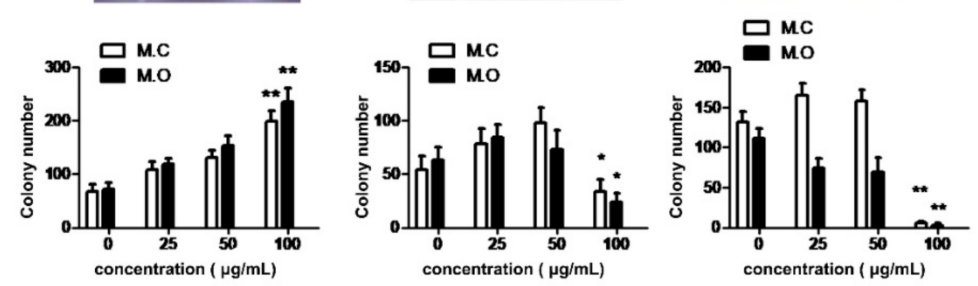

Figure 6. The effect of M. officinalis and M. citrifolia root extracts on cell growth. (A) Cell viability was evaluated by a CCK-8 assay kit. ${ }^{*} p<0.05$, compared to $24 \mathrm{~h}$ treatment group. (B) The colony formation ability of cells was detected through a colony formation assay. ${ }^{*} p<0.05$, ${ }^{* *} p<0.01$, compared with untreated cells. M.C: the methanol extract from M. citrifolia root. M.O: the methanol extract from M. officinalis root.

Numerous studies indicated that $M$. citrifolia extracts exhibited anticancer activity [23]. For instance, the leaf extract of $M$. citrifolia has an inhibitory effect on lung cancer through strengthening immune response [24]. Its fruit extracts induced apoptosis and suppressed migration in human liver and breast cancer cells [25]. In contrast, few publications report the antitumor activity of M. officinalis. Our results are consistent with previous reports that $M$. officinalis root extracts show poor cell cytotoxicity toward cancer cells, compared with $M$. citrifolia root extracts. 


\section{Materials and Methods}

\subsection{Instruments and Materials}

A Waters ACQUITY UPLC (Milford, MA, USA) coupled to a Xevo G2-XS Q-Tof (Milford, MA, USA) mass spectrometer was utilized. The ACQUITY UPLC HSS T3 RP-18 column $(100 \times 2.1 \mathrm{~mm}$, $1.8 \mu \mathrm{m}$ ) was purchased from Waters (Milford, MA, USA). Masslynx V4.1, UNIFI ${ }^{\circledR}$ and Progenesis QI were also from Waters (Milford, MA, USA). A precision scale (Secura513-1CN) was used (Sartorius Corporation, Germany).

Pall GHPAcrodisc ${ }^{\circledR}$ Syringe filters $(0.2 \mu \mathrm{m}, 13 \mathrm{~mm})$ were purchased from Pall (Pensacola, FL, USA). Disposable sterilized syringes $(1 \mathrm{~mL})$ and needles were purchased from Fenglin Medical Apparatus Corporation Ltd. (Jiangxi, China). Pipette tips $(1000 \mu \mathrm{L}, 200 \mu \mathrm{L}$ and $20 \mu \mathrm{L})$ were purchased from Labmed Biotech Corporation (USA). Centrifuge tubes were from KiRGEN (Atlanta, GA, USA). Vials (1.5 mL and $2 \mathrm{~mL}$ ) were purchased from Waters (Milford, MA, USA).

HPLC-grade methanol, acetonitrile, and water were from Fisher (Pittsburgh, PA, USA). Formic acid (LC/MS grade) was purchased from Merck Millipore (Darmstadt, Germany). ACS grade sodium hydroxide was obtained from Sigma-Aldrich (St. Louis, MO, USA). Distilled water was purchased from Watsons (Guangzhou, China). Lastly, ultrapure water was obtained through Milli-Q purification system (Merck Millipore, Darmstadt, Germany). Other solvents and reagents used were of analytical grade.

\subsection{Plant Materials}

The roots of M. officinalis (How.) and M. citrifolia (Linn.) were collected from the germplasm repository of tropical medicinal plants, Danzhou City, Ministry of Agriculture, Hainan Province, China, in November and December 2018. M. officinalis (How.) was introduced from Diaoluoshan, Lingshui Li Autonomous Count, Hainan Province, China. M. citrifolia (Linn.) was introduced from Xisha yongxing island, Hainan Province, China.

The identification of the voucher specimens was authenticated by Maoyuan Wang from the Tropical Crops Genetic Resources Institute, Chinese Academy of Tropical Agricultural Sciences. Specimens were then deposited in the Key Laboratory of Crop Gene Resources and Germplasm Enhancement in Southern China, Ministry of Agriculture, Hainan Haikou, China (No. B20181121 and B20181208). The plant materials were air-dried and ground for the extraction procedure.

\subsection{Sample Preparation}

The air-dried roots of $M$. officinalis and M. citrifolia were ground and passed through an 80-mesh sieve. Each powdered sample $(1.0 \mathrm{~g})$ was mixed with methanol $(50 \mathrm{~mL})$ and then sonicated for $1 \mathrm{~h}$ at a temperature of $40{ }^{\circ} \mathrm{C}$. The extract was subjected to centrifugation $\left(12,000 \mathrm{rpm}, 10{ }^{\circ} \mathrm{C}\right)$ for $10 \mathrm{~min}$. The supernatant was passed through a $0.2 \mu \mathrm{m}$ polytetrafluoroethylene (PTFE) syringe filter (VWR Bridgeport, PA, USA) and transferred to a $2 \mathrm{~mL}$ transparent vial [19].

\subsection{UPLC/Q-TOF-MS Detection Conditions}

\subsubsection{Chromatographic Conditions}

The separation was conducted on a Waters ACQUITY UPLC system with an ACQUITY UPLC HSS T3 C18 column and 0.1\% aqueous formic acid (A) and acetonitrile with $0.1 \%$ formic acid (B) was applied as the solvent system. The flow rate was $0.3 \mathrm{~mL} / \mathrm{min}$, and each sample solution was injected $1 \mu \mathrm{L}$. The column temperature was controlled at $40{ }^{\circ} \mathrm{C}$. Five injections each were performed for both, sample and the blank control. A linear gradient elution was used as follows: 0-4 min, 3-15\% B; 4-12 $\min , 15-60 \%$ B; 12-16 min, 60-80\% B; 16-18 $\mathrm{min}, 80-100 \%$ B; 18-21 min, 100-100\% B; 21-22 min, $100-3 \% \mathrm{~B} ; 22-25 \mathrm{~min}, 3-3 \% \mathrm{~B}$. 


\subsubsection{Mass Spectometry Conditions}

A Waters Xevo G2 QTOF equipped with Z-Spray electro spray ionization (ESI) source and MassLynx 4.1 was used for MS data acquisition. Negative ionization mode was applied to acquire data under a mass range of 50-1200 Da with a scan time of $0.2 \mathrm{~s}$ and detection time of $22 \mathrm{~min}$; furthermore, both low-energy (function 1) and high-energy (function 2) scan functions were used. The collision energy was $6 \mathrm{~V}$ for the low-energy scan function, and 10-45 V for the high-energy scan function. The capillary voltages were set at $2.0 \mathrm{kV}$, and the sampling cone voltage at $40 \mathrm{~V}$. The source and desolvation temperatures were $100{ }^{\circ} \mathrm{C}$ and $400{ }^{\circ} \mathrm{C}$, respectively. The desolvation gas flow rate was $600 \mathrm{~L} / \mathrm{h}$, while the cone gas flow rate was $50 \mathrm{~L} / \mathrm{h}$.

\subsection{Mass Data Processing and Analysis}

Progenesis QI software was utilized to convert the data of UPLC/Q-TOF-MS, i.e.,; then, the significant differential retention time-exact mass (RT-EM) pairs in the S-plots were picked and exported back into Progenesis QI for compound structural elucidation. The resultant data matrices were subsequently exported to EZinfo 2.0 software (MassLynx v4.1, Waters) for PCA (principal component analysis) and an orthogonal partial least squares-discriminant analysis (OPLS-DA) to examine differences in the samples. All variables obtained from UPLC-MS datasets were mean-centered and scaled to Pareto variance before subjection to PCA and OPLS-DA. The OPLS-DA score plots were depicted by the cross-validation parameter R2Y and Q2, which signify the total explained variation for the $\mathrm{X}$ matrix and the predictability of the model, respectively. The sum of squares of the PLS weights was evaluated by the value of VIP (variable importance in projection), showing the relative contribution of each $X$ variable in the model. The variables with VIP $>3$ were considered influential for the separation of samples in the score plots from the PLS-DA analysis [26,27].

\subsection{Chemical Composition and Biomarker Identification}

Multiple approaches were applied to identify chemical components and biomarkers, which include; comparison with retention times, accurate molecular ions, and characteristic fragment ions of reference compounds; reported data of the same compounds in the literature; online Traditional Chinese Medicine (TCM) Chinese [UNIFI1.7]; and ChemSpider [27].

\subsection{Cell Viability Assay}

Cells were planted into 96-well plates. Various concentrations of the extracts $(0.4,1.2,3.7,11.1$, 33.3 and $100 \mu \mathrm{g} / \mathrm{mL}$ ) were added into the culture media for $24 \mathrm{~h}, 48 \mathrm{~h}$, and $72 \mathrm{~h}$. At $1 \mathrm{~h}$ before each time point, a $10 \mu \mathrm{L}$ volume of CCK-8 reagent was added to each well. Optical density values at $450 \mathrm{~nm}$ were read with a microplate reader (Elx808, Bio Tek, Winooski, VT, USA) and analyzed with GraphPad Prism 7.0 software (GraphPad, San Diego, CA, USA).

\subsection{Cell Colony Formation Assay}

Cells were plated into 12-well plates in a triplicate manner. The root extracts of $M$. officinalis and M. citrifolia $(25,50$, and $100 \mu \mathrm{g} / \mathrm{mL})$ were added to the plates. Culture media were replaced every third day. After 10 days, the culture medium was discarded, and cells were washed by phosphate-buffered saline (PBS). Then, cells were fixed with $4 \%$ paraformaldehyde (Beyotime Biotechnology, Shanghai, China) for $30 \mathrm{~min}$, and stained with crystal violet (Beyotime) for $30 \mathrm{~min}$, followed by a wash with distilled water. The colonies were imaged by a HP scanner and counted under an inverted phase contrast microscope. 


\subsection{Data Analysis}

Cell experiments were performed in triplicate, and the results are presented as the mean value \pm standard error of the mean (SEM). Data were analyzed using GraphPad Prism 7.0 software (GraphPad, San Diego, CA, USA), and $\mathrm{p}<0.05$ was considered statistically significant.

\section{Conclusions}

In the present study, a total of 49 compounds were identified from the methanol extract of the root of M. officinalis and 39 compounds were identified from the methanol extract of the root of M. citrifolia by a UPLC-QTOF-MS/MS analysis. We found 25 ingredients that were present in both plants; they consisted of anthraquinones, triterpene, and iridoid glycosides. Using a QI analysis, we identified 9 components that could be used as chemical markers to distinguish the root extracts of M. officinalis from those of $M$. citrifolia introduced from Hainan Province. In addition, we found that M. officinalis and M. citrifolia exhibited no cell cytotoxicity towards normal cells, and even promoted the proliferation of normal liver cells. Our study suggests that $M$. officinalis and $M$. citrifolia roots have similarities in terms of chemical composition and biological activity. However, further research is needed to explore the feasibility and substitutability of the clinical application of M. officinalis and M. citrifolia.

Supplementary Materials: The following are available online. Figure S1: The propose fragmentation pathways of anthraquinones; Figure S2: The propose fragmentation pathways of anthraquinones.

Author Contributions: Funding acquisition, M.W. and Z.W.; Methodology, X.Y.; Project administration, Q.W.; Writing —original draft, Q.Y.; Writing—review \& editing, S.F. All authors have read and agreed to the published version of the manuscript.

Funding: This paper was supported by Financial Fund of the Ministry of Agriculture and Rural Affairs, P. R. of China (NFZX2018). Central Public-interest Scientific Institution Basal Research Fund for Chinese Academy of Tropical Agricultural Sciences (No. 1630032019021).

Conflicts of Interest: The authors declare that they have no conflict of interest.

\section{References}

1. Yoshikawa, M.; Yamaguchi, S.; Nishisaka, H.; Yamahara, J.; Murakami, N. Chemical constituents of Chinese natural medicine, Morindae Radix, the dried roots of Morinda officinalis How: Structures of morindolide and morofficinaloside. Chem. Pharm. Bull. 1995, 43, 1462-1465. [CrossRef] [PubMed]

2. Zhang, J.-H.; Xin, H.-L.; Xu, Y.-M.; Shen, Y.; He, Y.-Q.; Hsien-Yeh, H.; Lin, B.; Song, H.-T.; Liu, J.; Yang, H.-Y. Morinda officinalis How-A comprehensive review of traditional uses, phytochemistry and pharmacology. J. Ethnopharmacol. 2017, 213, 230-255. [PubMed]

3. Xia, T.; Dong, X.; Lin, L.; Jiang, Y.; Ma, X.; Xin, H.; Zhang, Q.; Qin, L. Metabolomics profiling provides valuable insights into the underlying mechanisms of Morinda officinalis on protecting glucocorticoid-induced osteoporosis. J. Pharm. Biomed. Anal. 2019, 166, 336-346. [CrossRef] [PubMed]

4. Kim, I.T.; Park, H.J.; Nam, J.H.; Park, Y.M.; Won, J.H.; Choi, J.; Choe, B.K.; Lee, K.T. In-vitro and in-vivo anti-inflammatory and antinociceptive effects of the methanol extract of the roots of Morinda officinalis. J. Pharm. Pharmacol. 2010, 57, 607-615. [CrossRef]

5. Soon, Y.; Tan, B.K.H. Evaluation of the hypoglycaemic antioxidant activities of Morinda officinalis in streptozotocin induced diabetic rats. Singap. Med. J. 2002, 43, 77-85.

6. Zhang, H.-L.; Li, J.; Li, G.; Wang, D.-M.; Zhu, L.-P.; Yang, D.-P. Structural characterization and anti-fatigue activity of polysaccharides from the roots of Morinda officinalis. Int. J. Biol. Macromol. 2009, 44, 257-261. [CrossRef]

7. Potterat, O.; Hamburger, M. Morinda citrifolia (Noni) fruit-phytochemistry, pharmacology, safety. Planta Med. 2007, 73, 191-199. [CrossRef]

8. Nayak, B.S.; Marshall, J.R.; Isitor, G.; Adogwa, A. Hypoglycemic and hepatoprotective activity of fermented fruit juice of Morinda citrifolia (Noni) in diabetic rats. Evid. Based Complement. Altern. Med. 2011, 2011, 875293. [CrossRef] 
9. Chan-Blanco, Y.; Vaillant, F.; Perez, A.M.; Reynes, M.; Brillouet, J.-M.; Brat, P. The noni fruit (Morinda citrifolia L.): A review of agricultural research, nutritional and therapeutic properties. J. Food Compos. Anal. 2006, 19, 645-654. [CrossRef]

10. Assi, R.A.; Darwis, Y.; Abdulbaqi, I.M.; Vuanghao, L.; Laghari, M. Morinda citrifolia (Noni): A comprehensive review on its industrial uses, pharmacological activities, and clinical trials. Arab. J. Chem. 2017, 10, 691-707. [CrossRef]

11. Krishnakumar, N.; Latha, P.; Suja, S.; Rajasekharan, S. A review on the ethnomedicinal, therapeutic and nutraceutical importance of "Noni" (Morinda citrifolia L.). IJMPNP 2015, 1, 1-14.

12. Deng, S.; West, B.J.; Palu, A.K.; Zhou, B.-N.; Jensen, C.J. Noni as an anxiolytic and sedative: A mechanism involving its gamma-aminobutyric acidergic effects. Phytomedicine (Jena) 2007, 14, 517-522. [CrossRef] [PubMed]

13. Palu, A.K.; Kim, A.H.; West, B.J.; Deng, S.; Jensen, J.; White, L. The effects of Morinda citrifolia L. (noni) on the immune system: Its molecular mechanisms of action. J. Ethnopharmacol. 2008, 115, 502-506. [CrossRef] [PubMed]

14. Wang, J.; Shi, J.; Wei, X.F.; Ren, X.H.; Li, X.W. Effect of Morinda officinalis on Kidney-Yang Deficiency Infertility Rats. J. Chin. Med. Mater. 2017, 40, 1826-1832.

15. Kamiya, K.; Tanaka, Y.; Endang, H.; Umar, M.; Satake, T. Chemical constituents of Morinda citrifolia fruits inhibit copper-induced low-density lipoprotein oxidation. J. Agric. Food Chem. 2004, 52, 5843-5848. [CrossRef]

16. Xu, Y.; Cai, H.; Cao, G.; Duan, Y.; Pei, K.; Tu, S.; Zhou, J.; Xie, L.; Sun, D.; Zhao, J. Profiling and analysis of multiple constituents in Baizhu Shaoyao San before and after processing by stir-frying using UHPLC/Q-TOF-MS/MS coupled with multivariate statistical analysis. J. Chromatogr. B 2018, 1083, 110-123. [CrossRef]

17. Aly, A.H.; Edrada-Ebel, R.; Wray, V.; Müller, W.E.; Kozytska, S.; Hentschel, U.; Proksch, P.; Ebel, R. Bioactive metabolites from the endophytic fungus Ampelomyces sp. isolated from the medicinal plant Urospermum picroides. Phytochemistry 2008, 69, 1716-1725. [CrossRef]

18. Lu, J.Q.; Sun, M.Q.; Zhang, H.G. Mechanisms of catalpol and jasminoidin by electrospray ionization mass spectrometry. Chin. Tradit. Herb. Drugs 2008, 7, 1011-1014.

19. Zhao, X.; Wei, J.; Yang, M. Simultaneous analysis of iridoid glycosides and anthraquinones in Morinda officinalis using UPLC-QqQ-MS/MS and UPLC-Q/TOF-MSE. Molecules 2018, 23, 1070. [CrossRef]

20. Zhai, H.-J.; Yu, J.-H.; Zhang, Q.; Liu, H.-S.; Zhang, J.-S.; Song, X.-Q.; Zhang, Y.; Zhang, H. Cytotoxic and antibacterial triterpenoids from the roots of Morinda officinalis var. officinalis. Fitoterapia 2019, 133, 56-61. [CrossRef]

21. Lu, X.; Zou, W.; Dong, Y.; Zhou, D.; Tong, X.; Dong, Z.; Liang, G.; Tang, L.; Liu, M. Anti-renal fibrosis effect of asperulosidic acid via TGF- $\beta 1 / \mathrm{smad} 2 / \operatorname{smad} 3$ and NF- $\mathrm{kB}$ signaling pathways in a rat model of unilateral ureteral obstruction. Phytomedicine 2019, 53, 274-285.

22. He, J.; Xianyuan, L.; Ting, W.; Yaqian, D.; Zheng, C.; Lan, T.; Menghua, L. Asperuloside and Asperulosidic Acid Exert an Anti-Inflammatory Effect via Suppression of the NF-kB and MAPK Signaling Pathways in LPS-Induced RAW 264.7 Macrophages. Int. J. Mol. Sci. 2018, 19, 2027. [CrossRef] [PubMed]

23. Brown, A.C. Anticancer Activity of Morinda citrifolia (Noni) Fruit: A Review. Phytother. Res. 2012, 26, 1427-1440. [CrossRef] [PubMed]

24. Lim, S.L.; Goh, Y.M.; Noordin, M.M.; Rahman, H.S.; Othman, H.H.; Bakar, N.A.A.; Mohamed, S. Morinda citrifolia edible leaf extract enhanced immune response against lung cancer. Food Funct. 2016, 7, 741-751. [CrossRef]

25. Huang, C.; Wei, Y.-X.; Shen, M.-C.; Tu, Y.-H.; Wang, C.-C.; Huang, H.-C. Chrysin, Abundant in Morinda citrifolia Fruit Water-EtOAc Extracts, Combined with Apigenin Synergistically Induced Apoptosis and Inhibited Migration in Human Breast and Liver Cancer Cells. J. Agric. Food Chem. 2016, 64, 4235-4245. [CrossRef] 
26. Lin, H.; Zhu, H.; Tan, J.; Wang, C.; Dong, Q.; Wu, F.; Wang, H.; Liu, J.; Li, P.; Liu, J. Comprehensive investigation on metabolites of Wild-Simulated American Ginseng Root based on Ultra-high Performance Liquid Chromatography-Quadrupole Time-Of-Flight Mass Spectrometry. J. Agric. Food Chem. 2019, 67, 5801-5819. [CrossRef]

27. Gu, R.; Rybalov, L.; Negrin, A.; Morcol, T.; Long, W.; Myers, A.K.; Isaac, G.; Yuk, J.; Kennelly, E.J.; Long, C. Metabolic Profiling of Different Parts of Acer truncatum from the Mongolian Plateau Using UPLC-QTOF-MS with Comparative Bioactivity Assays. J. Agric. Food Chem. 2019, 67, 1585-1597. [CrossRef]

Sample Availability: Samples of the compounds of Morinda officinalis and Morinda citrifolia are available from the authors.

(C) 2019 by the authors. Licensee MDPI, Basel, Switzerland. This article is an open access article distributed under the terms and conditions of the Creative Commons Attribution (CC BY) license (http://creativecommons.org/licenses/by/4.0/). 